Published in final edited form as:

Dysphagia. 2019 February ; 34(1): 129-137. doi:10.1007/s00455-018-9924-5.

\title{
Volumetric changes to the pharynx in healthy aging: Consequence for pharyngeal swallow mechanics and function
}

\author{
Sonja M Molfenter, PhD ${ }^{1}$, Charles Lenell ${ }^{1}$ [PhD Candidate], and Cathy L Lazarus, PhD $^{2,3}$ \\ ${ }^{1}$ Department of Communicative Sciences \& Disorders, NYU Steinhardt \\ ${ }^{2}$ THANC Foundation, Department of Otolaryngology Head \& Neck Surgery, Mount Sinai Beth \\ Israel \\ ${ }^{3}$ Department of Otolaryngology Head \& Neck Surgery, Icahn School of Medicine at Mount Sinai
}

\section{Abstract}

Purpose-Pharyngeal lumen volume is prone to increase as a consequence of pharyngeal muscle atrophy in aging. Yet, the impact of this on swallowing mechanics and function is poorly understood. We examined the relationship between pharyngeal volume and pharyngeal swallowing mechanics and function in a sample of healthy community-dwelling seniors.

Methods-Data was collected from 44 healthy seniors (21 male, mean age=76.9, SD=7.1). Each participant swallowed 9 boluses of barium $(3 \times 5 \mathrm{ml}$ thin, $3 \times 20 \mathrm{ml}$ thin, $3 \times 5 \mathrm{ml}$ nectar). Pharyngeal shortening, pharyngeal constriction, pyriform sinus and vallecular residue were quantified from lateral view videofluorosopic swallowing studies. Pharyngeal lumen volume was captured during an oral breathing task with acoustic pharyngometery. In addition, within-participant measures of strength and anthropometrics were collected. Four linear mixed effects regression models were run to study the relationship between pharyngeal volume and pharyngeal constriction, pharyngeal shortening, pyriform sinus residue, and vallecular residue while controlling for bolus condition, age, sex and posterior tongue strength.

Results-Increasing pharyngeal lumen volume was significantly related to worse constriction and vallecular residue. In general, larger and thicker boluses resulted in worse pharyngeal constriction and residue. Pharyngeal shortening was only significantly related to posterior tongue strength.

Conclusions-Our work establishes the utility of acoustic pharyngometry to monitor pharyngeal lumen volume. Increasing pharyngeal lumen volume appears to impact both pharyngeal swallowing mechanics and function in a sample of healthy, functional seniors.

\section{Keywords}

swallowing; deglutition; dysphagia; deglutition disorders; aging; pharynx; sarcopenia; atrophy; acoustic pharyngometry; pharyngeal constriction; pharyngeal shortening; residue

\section{INTRODUCTION}

The proportion of seniors in the US is projected to dramatically increase from $13 \%$ (2010) to over $20 \%$ (2030) [1]. The literature suggests that between $11-38 \%$ of healthy, community- 
dwelling seniors experience disruption to swallowing function [2-8] which can potentially disrupt their nutrition, hydration, pulmonary health and quality-of-life. When patients with dysphagia are admitted to the hospital, they experience a $40 \%$ longer length-of-stay than those without dysphagia; an increase that has been conservatively estimated to cost 547 million dollars per year [9]. This underscores the importance of identifying underlying mechanisms responsible for the development of dysphagia in the context of healthy aging and exploring ways to prevent and/or rehabilitate dysphagia in our rapidly-aging society.

Age-related atrophy (sarcopenia) of the swallowing musculature has been widely adopted as an explanation for the decline in swallow function observed with advancing age. This phenomenon has been established in the tongue [10-13] and the geniohyoid muscle [14] and there is also a body of evidence that suggests that the pharyngeal muscles experience agerelated atrophy [15-17]. Not surprisingly, there is complimentary evidence that pharyngeal lumen size (i.e. the volume of the pharynx) increases with aging. Comparison of neck MRIs between healthy younger and older women confirms that decreased muscle thickness occurs alongside increases in pharyngeal lumen volume [18]. The width of the pharynx during maximal expansion is significantly greater in elderly subjects compared with younger controls [15]. Finally, recent data using 320-row area detector computed tomography has shown hypopharyngeal volume increases with aging [19]. During swallowing, the pharyngeal muscles constrict and shorten the pharynx to prevent incomplete clearance of the bolus from the pharynx. Thus, pharyngeal muscle atrophy and expanding pharyngeal lumen size are possible explanations for the observation of post-swallow residue in older individuals [20-22].

Yet, the relationship between pharyngeal lumen volume and swallowing function in healthy aging is not well understood. This gap in our knowledge is partially attributable to the lack of a practical and non-invasive method for quantifying pharyngeal volume. In this study, we employ the use of Acoustic Pharyngometry (AP), a non-invasive, reliable and valid tool used in sleep and dental medicine [23-30]. AP works much like sonar, whereby sound waves are reflected within the oral and pharyngeal cavities to provide 3D measures of space. AP waveforms captured during breathing can be analyzed to calculate oral and pharyngeal length as well as oral and pharyngeal volume [31].

The primary goal of this research is to investigate the influence of pharyngeal volume on pharyngeal swallowing biomechanics (pharyngeal constriction and shortening) and postswallow residue (in the vallecular and pyriform sinuses) in the context of healthy aging. Our over-arching hypothesis was that increasing pharyngeal lumen volume would be associated with reductions in pharyngeal constriction, reductions in pharyngeal shortening, and increases in pharyngeal residue. Our research design allowed us to simultaneously investigate the influence of bolus condition (volume and viscosity), age and strength (tongue and hand grip) on pharyngeal biomechanics and pharyngeal residue in healthy aging seniors. 


\section{METHODS}

\section{Participants}

44 healthy seniors ( 21 male) over the age of 65 were recruited from local community centers. Exclusion criteria included a history of neurological disease/insult, significant head and neck surgery and/or chemoradiation to the head and neck. Our goal was to recruit a sample that would represent a range of sarcopenia/frailty; therefore, we screened tongue strength during recruitment events. We targeted both weak and strong individuals by enrolling a convenience sample of equal distribution of men and women with tongue strength over and under 40 kilopascals on the Iowa Oral Performance Instrument (IOPI) as this value appears to demarcate weak and strong older adults [32-34]. This study was approved by the local IRB and all subjects engaged in written consent to participate.

\section{Data Collection}

Data collection spanned two consecutive days. Day One was completed at the University Clinic and involved collecting participant demographics, interviews and oral motor sensory exams to ensure that participants met inclusion/exclusion criteria. Height was measured with a free-standing stadiometer. Next, various screening tasks were completed including EAT-10 [35], 3-ounce water swallow challenge [36] and the Frailty Index [37]. With the exception of hand grip strength (collected as a component of the Frailty Index), results from these screening tasks are not reported in this manuscript as they were collected for as part of a different hypothesis/aim. Maximal handgrip (left and right) was measured with a digital dynometer (Jamar) according to the operating manual. The highest value per participant was chosen to represent grip strength. Finally, anterior and posterior tongue strength were measured using the IOPI. Correct tongue bulb placement and compression location was first demonstrated by a trained research assistant and then practiced by the participant. The anterior position required the bulb placed on the tongue blade to be pressed against the alveolar ridge while the posterior position required the bulb to be placed on mid-dorsum and pressed against the mid-palate. After demonstration and practice, each participant conducted three tongue presses per position and the average score was used to represent tongue strength (see Table 1).

Day Two measures were collected at a local hospital and included Acoustic Pharyngometry (AP) and Videofluoroscopy (VF) protocols. AP was collected using the ECCOVISION® Acoustic Pharyngometer ${ }^{\mathrm{TM}}$ (Sleep Group Solutions). During AP acquisition, subjects were comfortably seated in a chair with their head and spine in a neutral position. The participant comfortably positioned the single-use mouthpiece connected to the wavetube in their oral cavity (lips outside the mouthpiece with teeth and tongue resting lightly in the corresponding guards/trays). Next, while the examiner held the wavetube parallel to the floor, the participant was instructed to complete the AP protocol breathing tasks: oral breathing, nasal breathing and modified Valsalva. These breathing tasks are required for post-processing within the ECCOVISION to measure pharyngeal volume. This process requires manually locating the velum (the lowest first trough from the nasal breathing task) and the glottis (the third trough of the modified Valsalva) to allow the examiner to calculate pharyngeal volume (between the velum and glottis) from the oral breathing waveform (see Figure 1). These 
procedures have been previously published [38], validated against MRI and CT [25, 30, 39] and established to be reliable [31].

The full VF protocol included 12 self-administered, uncued barium boluses (Varibar; Bracco Imaging) by $30 \mathrm{ml}$ medicine cups; however only the following 9 barium boluses are included in this analysis: $3 \times 5 \mathrm{ml}$ thin liquid, $3 \times 20 \mathrm{ml}$ thin liquid, $3 \times 5 \mathrm{ml}$ nectar thick liquid. Thin liquid barium was diluted $50 \%$ with water to create 'ultra-thin' liquid $(20 \% \mathrm{w} / \mathrm{v})$ which has been shown to be more sensitive to aspiration than the standard (40\%w/v) preparation [40]. Each bolus, measured via syringe, contained an additional $1 \mathrm{ml}$ more than the target volume based on research that confirms significant volumes of barium remain in the medicine cup after the swallow [41]. The order of bolus presentation was fixed to minimize aspiration risk (small boluses first) and to prevent contamination of thicker boluses (nectar last) to later trials. The VF data was collected on a GE Advantix digital fluoroscope (GE Healthcare) at a pulse rate of 30 pulses per second and captured at 30 frames per second on a Kay Pentax Digital Swallowing Workstation.

\section{Data Analysis}

All individual bolus clips were spliced out of the larger videofluoroscopic studies and randomized for blinded analysis by trained graduate and doctoral students using ImageJ [42] freeware. Variables of interest for the present study include measures of pharyngeal constriction, pharyngeal shortening and pharyngeal residue. Each bolus was also rated on the Penetration-Aspiration Scale [43] to confirm the overall level of functionality of these healthy seniors.

Pharyngeal constriction was captured by measuring the normalized maximum pharyngeal constriction area (MPCAn) on the frame of maximal constriction per previously published procedures [44]. Traditional pharyngeal constriction ratio measures [45] were not possible given that our data collection protocol did not include a $1 \mathrm{ml}$ bolus hold measure that is required to make this calculation. MPCAn is calculated by expressing the unobliterated area of the pharynx (represented by bolus or air) at maximal constriction during the swallow to the squared C2-4 length of the participant (which normalizes the area to the size of the participant). An example is provided in Figure 2A. When the pharynx is fully constricted, MPCAn is zero.

Pharyngeal shortening (PS) was originally measured in this dataset by tracking the peak position of the pyriform sinuses relative to the spine. However, this method yielded sub-par inter and intra rater reliability given that the bolus obstructs these structures at the height of the swallow. Therefore, building on research that confirms the strong mechanical correlations between PS and laryngeal elevation [46, 47], the peak superior position of the laryngeal air column (at the intersection of the vocal folds and the posterior trachea) was chosen as a proxy to pharyngeal shortening. Thus, the vertical position of the laryngeal air column at swallow peak was calculated from the anterior inferior corner of the $\mathrm{C} 4$ vertebra and normalized to the $\mathrm{C} 2-4$ length to control for subject size [48]. An example is provided in Figure 2B. 
Finally, quantitative measures of post-swallow residue were captured using the Normalized Residue Ratio Scale for the vallecula and piriform sinuses (NRRSv and NRRSp respectively) [49].

Twenty percent of all VF and AP data was selected at random and repeated by the original rater and also by a second trained research assistant to confirm adequate intra- and inter-rater reliability.

\section{Statistical Analysis}

Data was analyzed in IBM SPSS Statistics 24.0. Reliability was assessed using two-way mixed intraclass coefficients for consistency. Descriptive statistics were prepared for all dependent variables. Pearson correlations were calculated to assess the relationship between age and pharyngeal lumen volume. Variance Inflation Factor (VIF) was calculated to identify collinear predictors and demonstrated moderate collinearity (VIF $>3$ ) for several predictors. Variables were systematically removed according to hypothesized significance for the research design. VIFs were deemed appropriate $(<3)$ with a set of variables including pharyngeal volume, age and posterior tongue strength as predictors. Linear mixed effect regression models were run for each dependent variable (MPCAn, PS, NRRSv, NRRSp) while controlling for within subject repeated measures (swallow trial within bolus condition) and using a compound symmetry structure. Sex and bolus condition were included as fixed between-subjects factors, and pharyngeal volume, posterior tongue strength, and age were included as fixed within-subject factors. Post-hoc comparison of significant between subject main-effects controlled for Type I error via Sidak adjustments.

\section{RESULTS}

Table 1 summarizes participant demographics and physical characteristics. T-tests were conducted to compare demographic variables between men and women. Predictably, height, pharyngeal volume and hand grip strength were significantly greater in men compared with women. Note that all biomechanical swallowing parameters controlled for subject size (through normalization to C2-4 length).

Reliability results for all measured variables appear in Table 2. Only one score (inter-rater ICC for NRRSv) narrowly missed the 'excellent' category of ICC $>0.75$, and is thus categorized on the high end of the 'good' reliability range [50].

PAS scores are reported here to confirm the level of functionality of this sample of healthy seniors. PAS of 1 and 2 are considered normal [51, 52]. Of the 372 boluses administered in this analysis, only nine had PAS scores greater than 2 (eight scores of PAS $=3$, one score of PAS = 5). These nine scores come from 8 different participants. Therefore, the overall rate of penetration-aspiration was $2.4 \%$ of swallows $(9 / 372)$ and $18.1 \%$ of participants $(8 / 44)$.

Descriptive statistics for all dependent variables are reported by bolus condition in Table 3 .

\section{Pharyngeal Lumen Volume}

Correlations between pharyngeal lumen and each dependent variable are listed in Table 4. A significant main effect of pharyngeal volume was found using linear mixed effects 
regression models for both pharyngeal constriction $(\mathrm{F}=6.55, \mathrm{p}=0.015)$ and vallecular residue $(\mathrm{F}=7.75, \mathrm{p}=0.008)$ and trended toward significance for pyriform sinus residue $(\mathrm{F}=3.91$, $\mathrm{p}=0.055)$. For every unit $\left(1 \mathrm{~cm}^{3}\right)$ increase in pharyngeal volume, our data showed a 0.0013 increase in MCPAn (worse constriction with increasing volume) and a 0.0015 increase in NRRSv (worse vallecular residue with increasing volume). There was no significant effect of pharyngeal volume on pharyngeal shortening $(\mathrm{F}=1.17, \mathrm{p}=0.286)$.

\section{Tongue strength}

Pharyngeal shortening was the only dependent variable to have a main effect for posterior tongue strength $(\mathrm{F}=4.64, \mathrm{P}=0.037)$. For every unit increase in posterior tongue strength (1 $\mathrm{kPa}$ ), our data showed a $0.24 \% \mathrm{C} 2-4$ increase in pharyngeal shortening (improved shortening with stronger posterior tongue). There were no significant main effects of tongue strength for MPCAn or either NRRS score.

\section{Age and Sex}

Neither age nor sex was a significant independent predictor of any of the four dependent variables.

\section{Bolus Condition}

A significant main effect of bolus condition was found in each model (MCPAn: $\mathrm{F}=39.4$, $\mathrm{p}<0.000$; PS: $\mathrm{F}=5.63, \mathrm{p}=0.004$; NRRSv: $\mathrm{F}=10.25$, $\mathrm{p}<0.000$; NRRSp: $\mathrm{F}=3.06, \mathrm{p}=0.048$ ). Post-hoc pairwise comparisons elucidated several significantly different pairs (see Table 4, significant findings are bolded). In general, increasing volume and increasing viscosity resulted in more pharyngeal shortening, less constriction and worse residue.

\section{DISCUSSION}

In this study, we explored the relationship between pharyngeal lumen volume and pharyngeal swallowing in a sample of healthy community dwelling seniors. Our specific questions focused on the relationship between pharyngeal lumen volume and pharyngeal swallow mechanics (pharyngeal constriction and shortening) and the function associated with these actions (clearance of residue from the valleculae and the pyriform sinuses). Our findings confirmed that there is a significant negative relationship between the size of the pharyngeal lumen and pharyngeal constriction, as well as pharyngeal residue.

Significant efforts have focused on establishing that atrophy of swallowing musculature is responsible for the manifestation of age-related dysphagia, yet this notion has not been empirically investigated in the pharynx. A recent retrospective study of stroke patients by Sporns and colleagues [54] compared oropharyngeal muscle volume on CT and swallowing impairment on endoscopic evaluation of swallowing. Their analysis not only revealed significant reductions in muscle volume of the diagastric and temporal muscles by increasing age group but also by increasing dysphagia severity. In this study, we used noninvasive acoustic pharyngometry to quantify the size of the pharyngeal lumen and goldstandard VF to capture swallowing mechanics and function. Our overarching theory is that 
the expansion of the pharyngeal lumen due to muscular atrophy (observed in advancing age) is an underlying mechanism for the development of age-related dysphagia.

Specifically, we found that increasing pharyngeal lumen volume was associated with worse pharyngeal constriction. This finding is relatively intuitive - a larger space to constrict makes it more difficult to achieve full constriction. This finding aligns with previous studies showing that elderly subjects have greater unobliterated pharyngeal space during swallowing and worse pharyngeal constriction ratios compared to younger controls $[15,16]$. Taken together, these findings lend support to the notion that expanding pharyngeal lumen volume in aging could be a contributing factor in developing age-related dysphagia, especially given that this relationship was detectable in a healthy, functional community-dwelling cohort. Interestingly, recent work by Nativ-Zelter and colleagues [53] using simultaneous high resolution manometry and VF has demonstrated that healthy older individuals employ greater pharyngeal contraction compared with younger controls which could possibly be interpreted as a compensation for increasing pharyngeal lumen volume in aging.

This study has also confirmed a relationship between expanding pharyngeal lumen volume and increasing vallecular residue, and trended toward significance with increasing pyriform sinus residue. A function of pharyngeal constriction is to yield bolus clearing pressures [47] and thus the relationship between pharyngeal constriction and worse residue is physiologically expected and in line with our original hypotheses. This finding builds on work by Stokely and colleagues [55] which demonstrated that lower pharyngeal constriction (from 2D lateral VF) is associated with worse pharyngeal residue.

We failed to detect a significant relationship between pharyngeal shortening and pharyngeal volume. Pharyngeal shortening is achieved through the contraction of the longitudinal muscles of the pharynx including styplopharygeus, palatopharyngeas, and salpingopharygneas. Compared to the large, fan-shaped overlapping constrictor muscles, these relatively narrow longitudinal muscles of the pharynx may contribute less to the magnitude of pharyngeal lumen volume expansion observed in aging. Posterior tongue strength was significantly (and positively) associated with pharyngeal shortening, perhaps reflecting a relationship between the strength of these groups of oropharyngeal muscles.

When comparing pharyngeal constriction and post-swallow residue to a referent $5 \mathrm{ml}$ thin liquid bolus, participants demonstrated higher (worse) values for larger volumes and for thicker stimuli (for all comparisons except volume in the pyriforms). One could argue that these larger volumes and thicker viscosity conditions are 'more challenging' than the referent $5 \mathrm{ml}$ thin liquid. These results, from a narrow range of stimuli, demonstrate that even subtle adjustments in texture and volume can challenge the aging system. Yet interestingly, the reverse pattern exists for pharyngeal shortening. Higher values (more shortening) were achieved in the more challenging (larger volume, thicker viscosity) conditions. The participants in our sample appeared to modulate pharyngeal shortening for the bolus they were presented. Taken in combination with the fact that pharyngeal shortening appears to be unaffected by pharyngeal lumen volume increases, it may be the case that the longitudinal muscles of the pharynx are relatively spared in the healthy aging process. 
In addition to bolus condition, our research design allowed us to control for the variation attributable to age, sex, BMI, height, tongue strength (anterior and posterior), and hand grip strength. Because of high collinearity between variables, our final model only included posterior tongue strength, age, sex, and bolus condition. We did not find age or sex effects for any of the models that we tested. We included age in our analysis to determine if advancing age over 65 years old was a continuous predictor of swallowing biomechanics and/or function which is was not. It should be noted that all of the dependent measures we included are normalized during the measurement process. This controls for variation attributable to participant size, which can contribute to apparent sex-effects given that men are, on average, larger than women [56].

This sample is comprised of healthy seniors who volunteered to participate in a research study through recruitment from local community centers in a large urban neighborhood. Our distribution of penetration-aspiration scores is similar to those reported for other healthy older cohorts. However, we suspect that this dataset may have been vulnerable to a volunteer bias, in that our sample was relatively healthy and active compared with the average US senior. Indeed, frailty index measures [37] (collected for a different research question) revealed 18/44 participants scored 'non-frail', 24/44 scored 'pre-frail' and only 2/44 actually scored as 'frail'. Yet, despite this potential bias toward 'super' healthy seniors, the relationship between pharyngeal lumen volume and swallowing mechanics/function exists. We feel that this provides initial evidence that the reversal of pharyngeal muscle atrophy through specific exercises may be protective/preventative in healthy aging prior to the presentation of dysphagia. Further, this research establishes the utility of AP to monitor pharyngeal lumen volume in aging.

This study is not without its limitations. First, the interpretations are limited to our narrow range of stimuli and future work should expand this study to a larger range of textures and volumes. In addition, we acknowledge that the walls of the pharyngeal lumen are comprised of more than just the constrictor and longitudinal muscles of the pharynx. The anterior portion of the lumen is comprised of the base of the tongue as well as supraglottic larynx. We recognize that these structures are also prone to age-related changes that may contribute to increases in pharyngeal volume. The inclusion of posterior tongue strength was a deliberate choice to attempt to control for a portion of this variation. Further, twodimensional measures of pharyngeal constriction are limited in detecting degree of constriction. Future research should confirm the relationship between expanding pharyngeal lumen volume and worsening pharyngeal function using high resolution manometry. With respect to future studies, particularly significant contributions would be to establish a threshold of pharyngeal lumen volume that appears to result in functional impairment and to explore swallowing exercises that can reverse or counter-act pharyngeal atrophy.

\section{CONCLUSION}

In conclusion, we have confirmed that age-related pharyngeal atrophy in healthy seniors significantly (and negatively) impacts pharyngeal biomechanics and function. Specifically, we have demonstrated that larger pharyngeal lumen volume is associated with worse pharyngeal constriction and greater vallecular residue. These relationships were established 
in healthy aging individuals without complaint of dysphagia and thus exercises targeting pharyngeal muscle strengthening appear to hold promise as preventative and/or rehabilitative exercises to combat age-related dysphagia.

\section{Acknowledgments}

The authors would like to thank Erica Herzberg, Danielle Brates, Mehak Noorani, Emily Ottinger, Shelby Norman, Il Young Jung, Julie Bancroft, Wendy Liang, Chelsea Sandler, Marina Casale and Katrin Gabriel for their assistance during data collection and data analysis and Chuan-Ya Hsu and Ying Lu for statistical consultation.

Funding: This study was funded by NIH National Institute on Deafness and Other Communication Disorders 1R21DC015067.

\section{References}

1. Ortman, JM, Velkoff, VA, Hogan, H. Current Population Reports. U.S. Census Bureau; 2014. An Aging Nation: The Older Population in the United States; 25-1140.

2. Robbins J. Guest editorial: The current state of clinical geriatric dysphagia research. Journal of Rehabilitation Research and Development. 4:vii-ix.2002;

3. Chen P, Golub JS, Hapner ER, Johns MM III. Prevalence of perceived dysphagia and quality-of-life impairment in a geriatric population. Dysphagia. 1:1-6.2009;

4. Roy N, Stemple J, Merrill RM, Thomas L. Dysphagia in the elderly: Preliminary evidence of prevalence, risk factors, and socioemotional effects. Annals of Otology, Rhinology and Laryngology. 11:858-865.2007;

5. Bloem BR, Lagaay AM, van Beek W, Haan J, Roos RA, Wintzen AR. Prevalence of subjective dysphagia in community residents aged over 87. BMJ. 6726:721-722.1990;

6. Yang EJ, Kim MH, Lim J, Paik N. Oropharyngeal dysphagia in a community-based elderly cohort: The Korean longitudinal study on health and aging. J Korean Med Sci. 10:1534-1539.2013;

7. Kikawada M, Iwamoto T, Takasaki M. Aspiration and infection in the elderly. Drugs Aging. 2:115130.2005;

8. Holland G, Jayasekeran V, Pendleton N, Horan M, Jones M, Hamdy S. Prevalence and symptom profiling of oropharyngeal dysphagia in a community dwelling of an elderly population: a selfreporting questionnaire survey. Diseases of the Esophagus. 7:476-480.2011;

9. Altman KW, Yu G, Schaefer SD. Consequence of dysphagia in the hospitalized patient: impact on prognosis and hospital resources. Archives of Otolaryngology-Head \& Neck Surgery. 8:784789.2010;

10. Robbins J, Gangnon RE, Theis SM, Kays SA, Hewitt AL, Hind JA. The effects of lingual exercise on swallowing in older adults. J Am Geriatr Soc. 9:1483-1489.2005;

11. Nagai H, Russell JA, Jackson MA, Connor NP. Effect of aging on tongue protrusion forces in rats. Dysphagia. 2:116-121.2008;

12. Ota F, Connor NP, Konopacki R. Alterations in contractile properties of tongue muscles in old rats. Annals of Otology, Rhinology and Laryngology. 10:799-803.2005;

13. Butler SG, Stuart A, Leng X, Wilhelm E, Rees C, Williamson J, Kritchevsky SB. The relationship of aspiration status with tongue and handgrip strength in healthy older adults. Journals of Gerontology - Series A Biological Sciences and Medical Sciences. 4:452-458.2011;

14. Feng X, Todd T, Lintzenich CR, Ding J, Carr JJ, Ge Y, Browne JD, Kritchevsky SB, Butler SG. Aging-related geniohyoid muscle atrophy is related to aspiration status in healthy older adults. Journals of Gerontology - Series A Biological Sciences and Medical Sciences. 7:853-860.2013;

15. Leonard R, Kendall KA, McKenzie S. Structural displacements affecting pharyngeal constriction in nondysphagic elderly and nonelderly adults. Dysphagia. 2:133-141.2004;

16. Kendall KA, Leonard RJ. Pharyngeal constriction in elderly dysphagic patients compared with young and elderly nondysphagic controls. Dysphagia. 4:272-278.2001;

17. Aminpour S, Leonard R, Fuller SC, Belafsky PC. Pharyngeal wall differences between normal younger and older adults. ENT: Ear, Nose \& Throat Journal. 4:1-4.2011; 
18. Molfenter SM, Amin M, Branski RC, Brumm J, Hagiwara M, Roof S, Lazarus CL. Age-related changes in pharyngeal lumen size: A retrospective MRI analysis. Dysphagia. 3:321-327.2015;

19. Inamoto Y, Saitoh E, Okada S, Kagaya H, Shibata S, Baba M, Onogi K, Hashimoto S, Katada K, Wattanapan P. Anatomy of the larynx and pharynx: effects of age, gender and height revealed by multidetector computed tomography. J Oral Rehabil. 9:670-677.2015;

20. Dejaeger E, Pelemans W, Ponette E, Joosten E. Mechanisms involved in postdeglutition retention in the elderly. Dysphagia. 2:63-67.1997;

21. Frederick MG, Ott DJ, Grishaw EK, Gelfand DW, Chen MYM. Functional abnormalities of the pharynx: A prospective analysis of radiographic abnormalities relative to age and symptoms. Am $\mathrm{J}$ Roentgenol. 2:353-357.1996;

22. Rofes L, Arreola V, Romea M, Palomera E, Almirall J, Cabré M, Serra-Prat M, Clavé P. Pathophysiology of oropharyngeal dysphagia in the frail elderly. Neurogastroenterology and Motility. 8:851-858+e230.2010;

23. Kamal I. Test-retest validity of acoustic pharyngometry measurements. Otolaryngology - Head and Neck Surgery. 2:223-228.2004;

24. Brooks LJ, Byard PJ, Fouke JM, Strohl KP. Reproducibility of measurements of upper airway area by acoustic reflection. J Appl Physiol. 6:2901-2905.1989;

25. Marshall I, Maran NJ, Martin S, Jan MA, Rimmington JE, Best JJK, Drummond GB, Douglas NJ. Acoustic reflectometry for airway measurements in man: Implementation and validation. Physiol Meas. 2:157-169.1993;

26. Hoffstein V, Fredberg JJ. The acoustic reflection technique for non-invasive assessment of upper airway area. European Respiratory Journal. 5:602-611.1991;

27. Fredberg JJ, Wohl MEB, Glass GM, Dorkin HL. Airway area by acoustic reflections measured at the mouth. Journal of Applied Physiology Respiratory Environmental and Exercise Physiology. 5:749-758.1980;

28. Bradley TD, Brown IG, Grossman RF, Zamel N, Martinez D, Phillipson EA, Hoffstein V. Pharyngeal size in snorers, nonsnorers, and patients with obstructive sleep apnea. N Engl J Med. 21:1327-1331.1986;

29. Viviano JS. Acoustic reflection: Review and clinical applications for sleep-disordered breathing. Sleep and Breathing. 3:129-149.2002;

30. D’Urzo AD, Rubinstein I, Lawson VG, Vassal KP, Rebuck AS, Slutsky AS, Hoffstein V. Comparison of glottic areas measured by acoustic reflections vs. computerized tomography. J Appl Physiol. 1:367-370.1988;

31. Molfenter SM. The Reliability of Oral and Pharyngeal Dimensions Captured with Acoustic Pharyngometry. Dysphagia. 4:555-559.2016;

32. Nicosia MA, Hind JA, Roecker EB, Carnes M, Doyle J, Dengel GA, Robbins J. Age effects on the temporal evolution of isometric and swallowing pressure. J Gerontol A Biol Sci Med Sci. 11:M634-40.2000;

33. Youmans SR, Youmans GL, Stierwalt JA. Differences in tongue strength across age and gender: is there a diminished strength reserve? Dysphagia. 1:57-65.2009;

34. Stierwalt JA, Youmans SR. Tongue measures in individuals with normal and impaired swallowing. American journal of speech-language pathology. 2:148-156.2007;

35. Belafsky PC, Mouadeb DA, Rees CJ, Pryor JC, Postma GN, Allen J, Leonard RJ. Validity and reliability of the eating assessment tool (EAT-10). Annals of Otology, Rhinology and Laryngology. 12:919-924.2008;

36. DePippo KL, Holas MA, Reding MJ. Validation of the 3-oz water swallow test for aspiration following stroke. Arch Neurol. 12:1259-1261.1992;

37. Fried LP, Tangen CM, Walston J, Newman AB, Hirsch C, Gottdiener J, Seeman T, Tracy R, Kop WJ, Burke G, McBurnie MA, Cardiovascular Health Study Collaborative Research Group. Frailty in older adults: evidence for a phenotype. J Gerontol A Biol Sci Med Sci. 3:M146-56.2001;

38. Vorperian HK. VTLab Acoustic Pharyngometry (APh) Protocol: Data Collection (Part I) \& Data Analysis (Part II) 09/09. 2013 
39. D’Urzo AD, Lawson VG, Vassal KP, Rebuck AS, Slutsky AS, Hoffstein V. Airway area by acoustic response measurements and computerized tomography. Am Rev Respir Dis. 2:392395.1987;

40. Fink TA, Ross JB. Are we testing a true thin liquid? Dysphagia. 3:285-289.2009;

41. Molfenter SM, Steele CM. Variation in temporal measures of swallowing: Sex and volume effects. Dysphagia. 2:226-233.2013;

42. Schneider CA, Rasband WS, Eliceiri KW. NIH Image to ImageJ: 25 years of image analysis. Nature methods. 7:671.2012;

43. Rosenbek JC, Robbins JA, Roecker EB, Coyle JL, Wood JL. A penetration-aspiration scale. Dysphagia. 2:93-98.1996;

44. Stokely SL, Peladeau-Pigeon M, Leigh C, Molfenter SM, Steele CM. The relationship between pharyngeal constriction and post-swallow residue. Dysphagia. 30:349-356.2015; [PubMed: 25920993]

45. Leonard R, Rees CJ, Belafsky P, Allen J. Fluoroscopic Surrogate for Pharyngeal Strength: The Pharyngeal Constriction Ratio (PCR). Dysphagia. 1:13-17.2011;

46. Olsson R, Castell J, Johnston B, Ekberg O, Castell DO. Combined videomanometric identification of abnormalities related to pharyngeal retention. Acad Radiol. 5:349-354.1997;

47. Kahrilas PJ, Logemann JA, Lin S, Ergun GA. Pharyngeal clearance during swallowing: A combined manometric and videofluoroscopic study. Gastroenterology. 1:128-136.1992;

48. Molfenter SM, Hsu CY, Lu Y, Lazarus CL. Alterations to Swallowing Physiology as the Result of Effortful Swallowing in Healthy Seniors. Dysphagia. :1-9.2018 [PubMed: 28819914]

49. Pearson WG Jr, Molfenter SM, Smith ZM, Steele CM. Image-based measurement of post-swallow residue: The normalized residue ratio scale. Dysphagia. 2:167-177.2013;

50. Fleiss, JL. The design and analysis of clinical experiments. New York: Wiley; 1986.

51. Allen JE, White CJ, Leonard RJ, Belafsky PC. Prevalence of penetration and aspiration on videofluoroscopy in normal individuals without dysphagia. Dysphagia. 4:347-348.2010;

52. Daggett A, Logemann J, Rademaker A, Pauloski B. Laryngeal penetration during deglutition in normal subjects of various ages. Dysphagia. 4:270-274.2006;

53. Nativ-Zeltzer N, Logemann JA, Zecker SG, Kahrilas PJ. Pressure topography metrics for highresolution pharyngeal-esophageal manofluorography-a normative study of younger and older adults. Neurogastroenterol Motil. 5:721-731.2016;

54. Sporns PB, Muhle P, Hanning U, Suntrup-Krueger S, Schwindt W, Eversmann J, Warnecke T, Wirth R, Zimmer S, Dziewas R. Atrophy of swallowing muscles is associated with severity of dysphagia and age in patients with acute stroke. Journal of the American Medical Directors Association. 2017 Jul 1.18(7):635-e1.

55. Stokely S, Molfenter SM, Leigh C, Peladeau-Pigeon M, Steele CM. The relationship between pharyngeal constriction and post-swallow residue. Abstract, Dysphagia Research Society Meeting. Dysphagia. 6:737.2014;

56. Molfenter SM, Steele CM. Use of an anatomical scalar to control for sex-based size differences in measures of hyoid excursion during swallowing. Journal of Speech, Language, and Hearing Research. 3:768-778.2014; 


\section{Compliance with Ethical Standards}

Ethical approval: All procedures performed in studies involving human participants were in accordance with the ethical standards of the institutional and/or national research committee and with the 1964 Helsinki declaration and its later amendments or comparable ethical standards. Informed consent: Informed consent was obtained from all individual participants included in the study. 


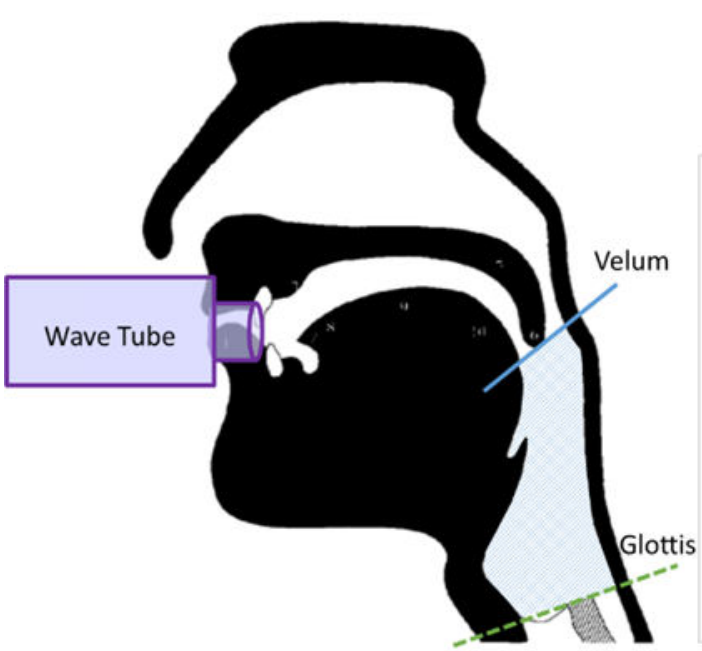

Pharyngeal Volume

Oral breathing $\left(\mathrm{cm}^{3}\right)$

- Nasal breathing Modified valsalva

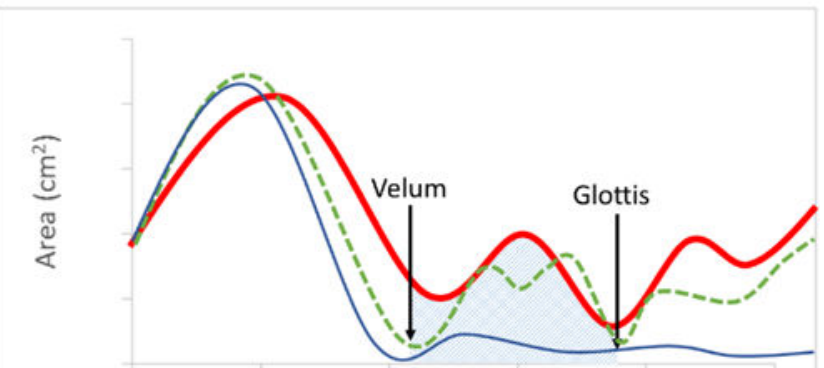

Length $(\mathrm{cm})$

Figure 1.

Schematic of process for capturing pharyngeal volume using Acoustic Pharyngometry. 


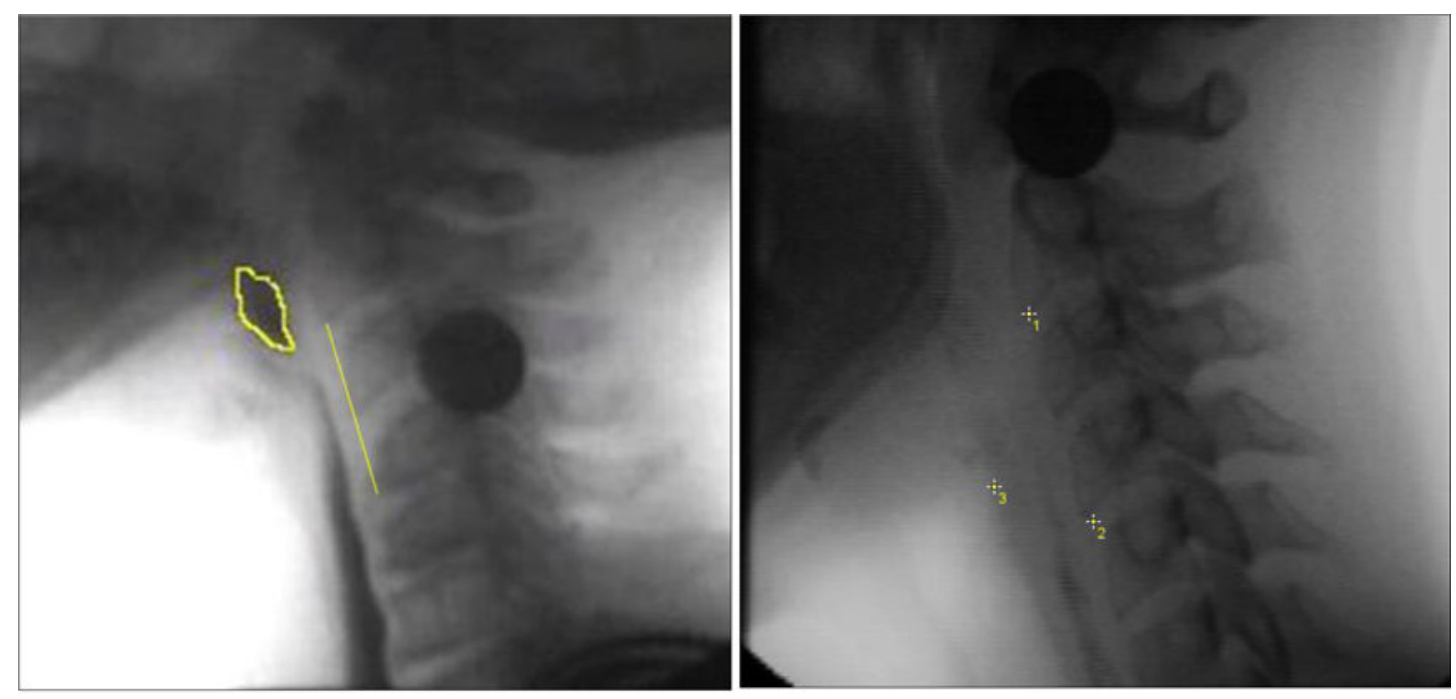

A

B

Figure 2.

A. Pharyngeal constriction measured as pixels of unobliterated pharyngeal space at maximal pharyngeal constriction, expressed as a function of the C2-4 distance squared. B. Pharyngeal shortening measured by expressing peak laryngeal position (point 3) from C4 (point 2) in a participant-defined coordinate system (Y axis through points 1 and 2). 

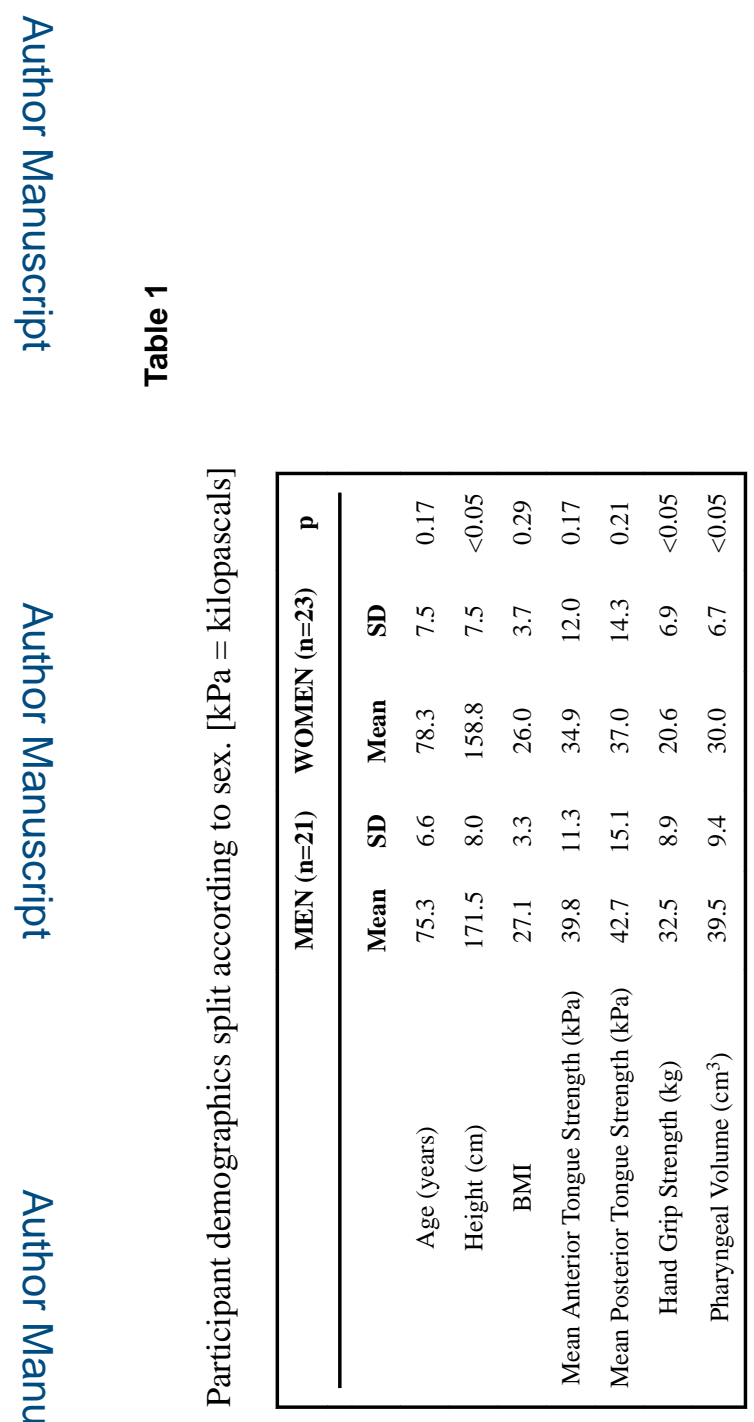

Dysphagia. Author manuscript; available in PMC 2020 February 01. 


\section{Table 2}

Reliability Results. [ICC = intraclass correlation coefficient, $\mathrm{CI}=$ confidence interval, $\mathrm{MPCAn}=$ Maximum pharyngeal constriction area normalized, $\mathrm{C} 2-4=\%$ of the distance of the second to fourth cervical vertebrae, NRRS $=$ normalized residue ratio scale]

\begin{tabular}{|ccccc|}
\hline & \multicolumn{2}{c}{ INTRA RATER } & \multicolumn{2}{c|}{ INTER RATER } \\
& ICC & $\mathbf{9 5 \%}$ CI & ICC & 95\% CI \\
\hline Pharyngeal Constriction (MPCAn) & 0.82 & $(0.73-0.88)$ & 0.76 & $(0.64-0.84)$ \\
Pharyngeal Shortening (\%C2-4) & 0.93 & $(0.90-0.95)$ & 0.93 & $(0.89-0.95)$ \\
Pharyngeal Volume (cm $\left.{ }^{3}\right)$ & 0.92 & $(0.66-0.98)$ & 0.96 & $(0.83-0.99)$ \\
Penetration Aspiration Scale & 0.88 & $(0.83-0.92)$ & 0.86 & $(0.79-0.90)$ \\
Vallecular Residue (NRRSv) & 0.89 & $(0.83-0.93)$ & 0.74 & $(0.61-0.82)$ \\
Piriform Sinus Residue (NRRSp) & 0.97 & $(0.96-0.98)$ & 0.86 & $(0.79-0.91)$ \\
\hline
\end{tabular}




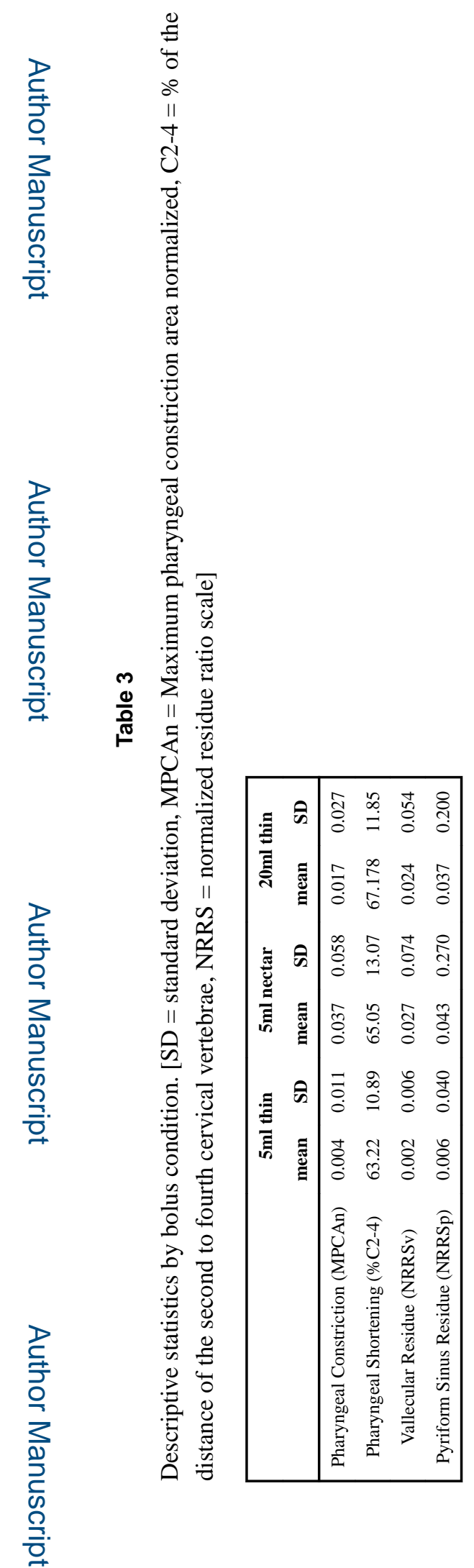

Dysphagia. Author manuscript; available in PMC 2020 February 01. 


\section{Table 4}

Pearson correlations between pharyngeal lumen volume and each dependent variable. [MPCAn = maximum pharyngeal constriction area normalized, C2-4 $=\%$ of the distance of the second to fourth cervical vertebrae, NRRS $=$ normalized residue ratio scale]

\begin{tabular}{|ccc|}
\hline & Correlation with Pharyngeal Volume & $\boldsymbol{p}$ \\
\hline Pharyngeal Constriction (MPCAn) & $\mathbf{0 . 2 9}$ & $<\mathbf{0 . 0 0 1}$ \\
Pharyngeal Shortening (\%C2-4) & 0.08 & 0.115 \\
Vallecular Residue (NRRSv) & $\mathbf{0 . 2 4}$ & $<\mathbf{0 . 0 0 1}$ \\
Pyriform Sinus Residue (NRRSp) & $\mathbf{0 . 1 6}$ & $\mathbf{0 . 0 0 2}$ \\
\hline
\end{tabular}




\section{Table 5}

Summary of bolus condition post-hoc comparisons for each dependent variable. Bolded values are significant. $[\mathrm{MPCAn}=$ Maximum pharyngeal constriction area normalized, C2-4 $=\%$ of the distance of the second to fourth cervical vertebrae, NRRS = normalized residue ratio scale]

\begin{tabular}{|cccc|}
\hline & & Volume & Viscosity \\
\cline { 2 - 4 } & & 5ml thin vs 20ml thin & 5ml thin vs 5ml nectar \\
\hline \multirow{2}{*}{ Pharyngeal Constriction (MPCAn) } & mean difference & $\mathbf{- 0 . 0 1 1}$ & $\mathbf{- 0 . 3 1 0}$ \\
& p value & $\mathbf{0 . 0 0 5}$ & $<\mathbf{0 . 0 0 1}$ \\
\hline \multirow{2}{*}{ Pharyngeal Shortening (\%C2-4) } & mean difference & $\mathbf{- 3 . 7 9 0}$ & -1.832 \\
& p value & $\mathbf{0 . 0 0 3}$ & 0.256 \\
\hline \multirow{2}{*}{ Vallecular Residue (NRRSv) } & mean difference & $\mathbf{- 0 . 0 2 1}$ & $\mathbf{- 0 . 0 2 5}$ \\
& p value & $\mathbf{0 . 0 0 1}$ & $<\mathbf{0 . 0 0 1}$ \\
\hline \multirow{2}{*}{ Pyriform Sinus Residue (NRRSp) } & mean difference & -0.028 & $\mathbf{- 0 . 0 3 8}$ \\
& p value & 0.237 & $\mathbf{0 . 0 5 0}$ \\
\hline
\end{tabular}

